\title{
Does the wearing of masks change voice and speech parameters?
}

\author{
R. Gama ${ }^{1,2}$ (D) Maria Eugénia Castro ${ }^{2} \cdot$ Julie Titske van Lith-Bij| $^{3} \cdot$ Gauthier Desuter $^{1}$
}

Received: 27 July 2021 / Accepted: 10 September 2021 / Published online: 22 September 2021

(c) The Author(s), under exclusive licence to Springer-Verlag GmbH Germany, part of Springer Nature 2021

\begin{abstract}
Purpose The authors aim to review available reports on the potential effects of masks on voice and speech parameters. Methods A literature search was conducted using MEDLINE and Google Scholar databases through July 2021. Several targeted populations, mask scenarios and methodologies were approached. The assessed voice parameters were divided into self-reported, acoustic and aerodynamic.

Results It was observed that the wearing of a face mask has been shown to induce several changes in voice parameters: (1) self-reported-significantly increased vocal effort and fatigue, increased vocal tract discomfort and increased values of voice handicap index (VHI) were observed; (2) acoustics-increased voice intensity, altered formants frequency (F2 and F3) with no changes in fundamental frequency, increased harmonics-to-noise ratio (HNR) and increased mean spectral values in high-frequency levels (1000-8000 Hz), especially with KN95 mask; (3) aerodynamics-maximum phonatory time was assessed in only two reports, and showed no alterations.

Conclusion Despite the different populations, mask-type scenarios and methodologies described by each study, the results of this review outline the significant changes in voice characteristics with the use of face masks. Wearing a mask shows to increase the perception of vocal effort and an alteration of the vocal tract length and speech articulatory movements, leading to spectral sound changes, impaired communication and perception. Studies analyzing the effect of masks on voice aerodynamics are lacking. Further research is required to study the long-term effects of face masks on the potential development of voice pathology.
\end{abstract}

Keywords Mask $\cdot$ Voice $\cdot$ Phonation $\cdot$ Acoustics $\cdot$ COVID-19 $\cdot$ Health personnel

\section{Introduction}

The widespread use of face masks, for both essential and professional activities, was a non-pharmacological intervention imposed by the ongoing global COVID-19 pandemic, to contain the transmission of the disease.

Previous studies showed that face masks have voice attenuation effects, and can work as an acoustic filter for speech,

R. Gama

ritarocha@campus.ul.pt

1 Voice and Swallowing Clinic, Department of Otolaryngology Head and Neck Surgery, Cliniques Universitaires Saint-Luc, 10 avenue Hippocrate, 1200 Brussels, Belgium

2 Department of Otorhinolaryngology Head and Neck Surgery, Vila Nova de Gaia/Espinho Hospital Center, Porto, Portugal

3 Department of Otorhinolaryngology, Flevoziekenhuis, Almere, The Netherlands especially in high frequencies [1]. This effect makes it harder for the listener to understand mask wearer's words, especially in noisy environments or if the listener has a hearing impairment [2]. However, we must considerer mask-wearing as a challenge also for the speaker, as he must overcome the mask visual and acoustic barrier and be able to communicate and be understood. Because face masks are placed over the nose and mouth, speech and voice characteristics may have to suffer important changes, both consequential and adaptive, inevitably leading to increased effort by the speaker in an attempt to be heard. It is hypothesized that this increased effort can significantly affect both intrinsic and extrinsic laryngeal muscles, leading to misuse vocal behaviors, in such a way that the prolonged mask-wearing can represent a potential health risk for vocal disorders [3].

Given the current data on the COVID-19 pandemic, we must assume that mask use is to be continued for a long time; therefore, it is becoming increasingly important to identify the consequences of its prolonged use on voice 
health, to design and implement preventive measures that could help mask wearers to improve vocal use. Because of the scarcity of scientific evidence, a state of art review will help to achieve a better understanding of mask and voice interaction. Till the moment of submission, this report presents the first literature review on the subject.

The authors aim to detect, analyze, interpret and summarize the potential effects of face masks on voice assessment parameters.

\section{Methods}

Articles on the subject were searched in MEDLINE and Google Scholar databases, from its inception through July 2021. The steps of the data collection process are outlined in Fig. 1.

The MEDLINE query used by the authors was: ["masks" [mesh] OR "face-masks"[tiab] OR "Covid19" [mesh] OR "health personnel" [mesh] OR "N95 respirators" [mesh] OR "mask-wearing" [tiab] AND "voice" [mesh] OR "voice changes" [tiab] OR "acoustic voice analysis [tiab]" OR "vocal assessment" [tiab]\}.

In total, 27 articles were considered. Inclusion criteria were: studies on mask effects on voice assessment, performed through one or more of the following parameters-perceptual, acoustic, aerodynamic and subjective \{ according to the European Laryngological Society (ELS) guideline [4]\}; articles written in English, French, Spanish, Italian, or Portuguese language; full-text articles; human studies.

After abstract processing, 16 full-text articles were assessed for eligibility. Exclusion criteria included studies performed in pathologic voices; non clarified and dubious methodology; studies focused on speech intelligibility rather than voice parameters. All references of the included full-text articles were reviewed to guarantee full-literature cover on the subject.

Lastly, after the necessary exclusions, nine studies were included in the qualitative analysis.
Fig. 1 Flow diagram showing the steps of data collection process

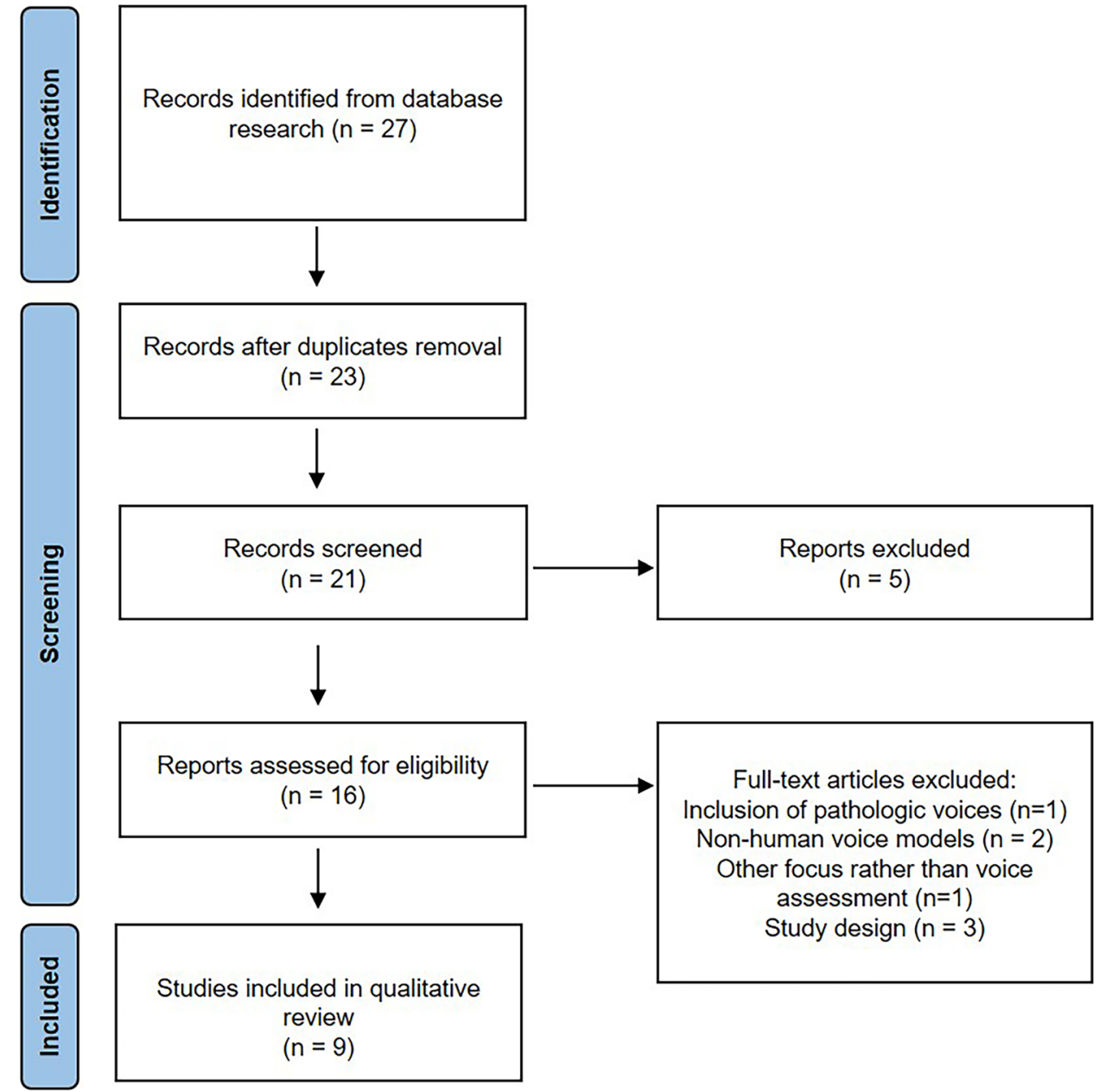




\section{Results}

The targeted population, the different mask scenarios as well as the main voice assessment parameters analyzed in each study are discriminated in Table 1 .

Selection and information bias, as well as the lack of a uniform methodology and reporting system made it impossible to perform a formal systematic review according to PRISMA standards. All the articles were observational studies, published during the last twelve months. A total of 1005 people were assessed for voice changes. Of the nine selected articles, six [5, 7, 8, 10, 11, 13] outlined acoustic parameters, four $[6,9,11,12]$ described self-reported vocal characteristics, and only two $[8,10]$ assessed voice aerodynamics. None of them described all three vocal domains, neither perceptual voice evaluation. The videostroboscopic assessment was not performed in any of the reviewed studies.

\section{Self-reported voice assessment}

All studies analyzing self-reported voice parameters were conducted through the use of several questionnaires, via online or presential mode, essentially comparing self-perceived voice changes between two scenarios: wearing and not wearing a face mask (most studies did not discriminate the type of mask being used), including healthcare workers and healthy volunteers from the general population, in both essential and professional activities. Most studies analyzed healthcare workers and other groups who use protective face mask during their working hours.

To analyze the self-perception of vocal effort and voicebreathing coordination while using face masks, questionnaires were applied, answered using a 5 or 6-point Likert Scale between zero (never) and 5-6 (always), as well as VAS (Visual Analog Scale) ratings [11] and VHI (Voice Handicap Index). [12] A significant increase in vocal effort perception, communication difficulties and a significantly increased difficulty in voice-breathing coordination with face masks were outlined in all the studies assessing selfreported voice changes. [6, 9-12] Increased values of VHI were observed mainly due to emotional and psychosocial voice and speech difficulties, with the particular case of health care workers in whom it was even higher after a long work-day and/or after working in Intensive Care Units (ICU) [12]. Besides, significantly higher scores of vocal fatigue symptoms in mask-wearing groups were verified, $[6,9,11]$ mostly tiredness, voice impairment and avoidance of voice use [6].

Vocal tract discomfort while wearing a face mask was assessed through vocal tract discomfort scale (VTDS), which includes the terms "burning", "tight", "dry", "aching", "tickling", "sore", "irritable" and "lump in the throat". [6,9]. The majority of individuals felt moderate to severe dryness, mild to moderate throat clearing, moderate to severe sensation of a lump in the throat and moderate to severe short breathiness while speaking with a face mask, [9] with a statistically significant difference between wearing and not-wearing mask groups in one of the studies $(p<0,001)[6]$.

Furthermore, studies also pointed out that speech intelligibility was significantly impaired with the use of face masks $[6,9,11]$.

\section{Acoustics}

The studies analyzing acoustic voice parameters were done in person, through the performance of speeching tasks by the participants, under different mask scenarios.

Overall, when comparing mask and no-mask scenarios (regardless of the type of mask), it was observed a significant increase in vocal intensity while wearing a face mask in all $[5,7,8,11]$ but two of the six studies performing acoustic voice evaluation $[10,13]$.

The harmonics-to-noise ratio (HNR) values were significantly increased with the wearing of a mask, either a surgical or a KN95, when compared to the no-mask condition $[8,13]$; it was also increased after a mask-wearing workday comparing with the pre-workday value [11]. However, cepstral peak prominence (CPP) showed none or minimally changes with mask-wearing in all studies $[5,7,8,13]$.

Fundamental frequency (F0) values did not seem to be significantly changed by the wearing of a mask $[5,7,8,10]$, although a significant decrease in relative fundamental frequency (RFF) offset ten was observed in mask wearers after a workday $(p=0,001)$, especially when wearing KN95 [11].

Some studies also measured formants frequency F1, F2 and F3 [5, 8]. Formant frequency F2 showed to be altered in Joshi et al. [5] decreasing in males with KSF scenario (face shield over surgical mask and KN95), and increased in females wearing a surgical mask when comparing with other mask-type scenarios. In another study, F2 showed increasing trends (although not significant) and F3 showed to be significantly decreased with masks comparing with no-mask scenario $(p=0,004)$ [8].

Jitter and shimmer values showed no significant changes while using a mask in most studies [7, 10], except for one of them that showed significantly decreased values of both jitter and shimmer when comparing with no-mask scenario [8].

Some speech and sound characteristics also showed to be modified: mask-type impacted the acoustic power distribution in frequencies above $3000 \mathrm{~Hz}$ for KN95 mask, and above $5000 \mathrm{~Hz}$ in surgical and cloth masks, comparing with no-mask status [7]. This impact was not observed 


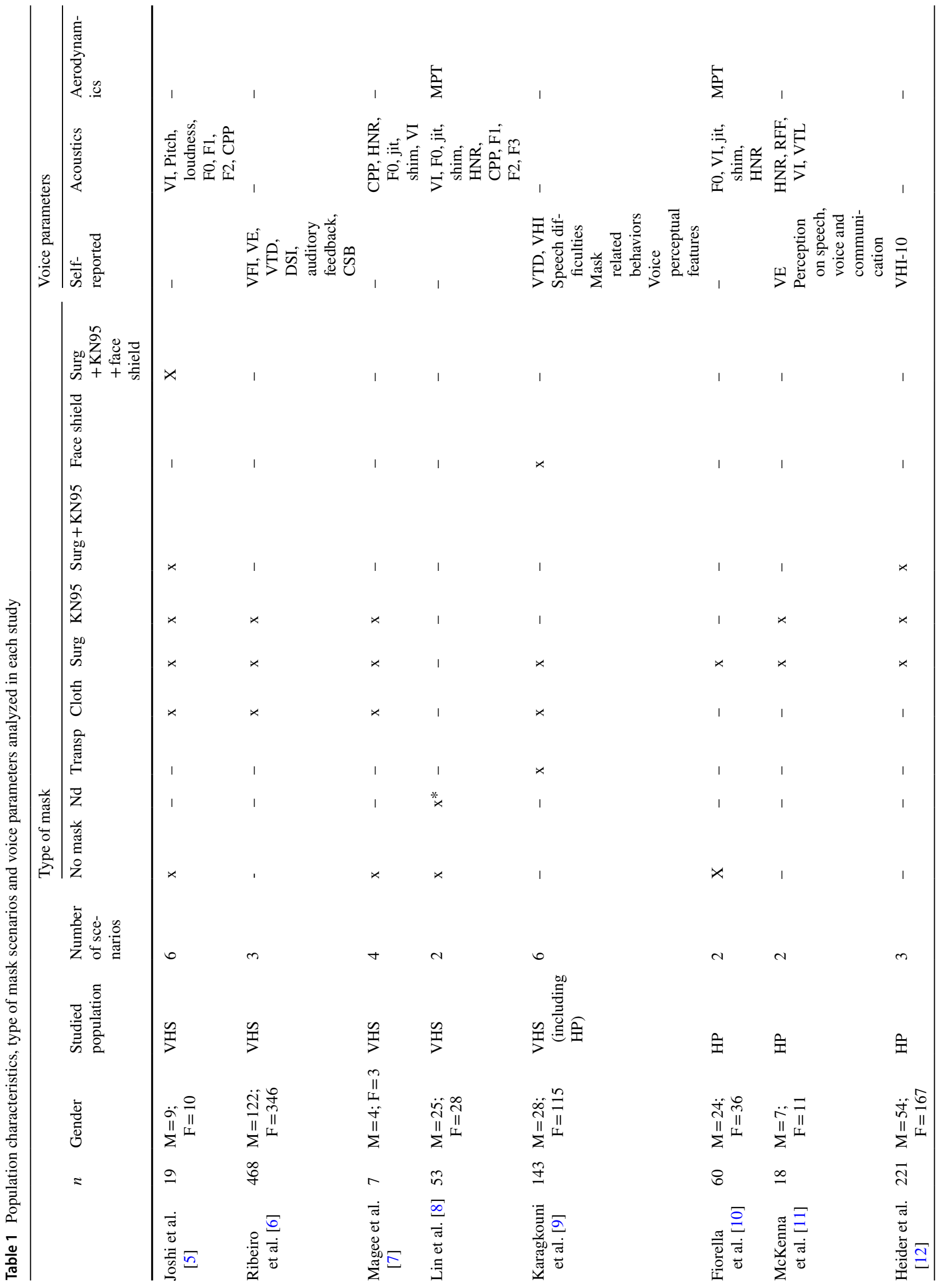




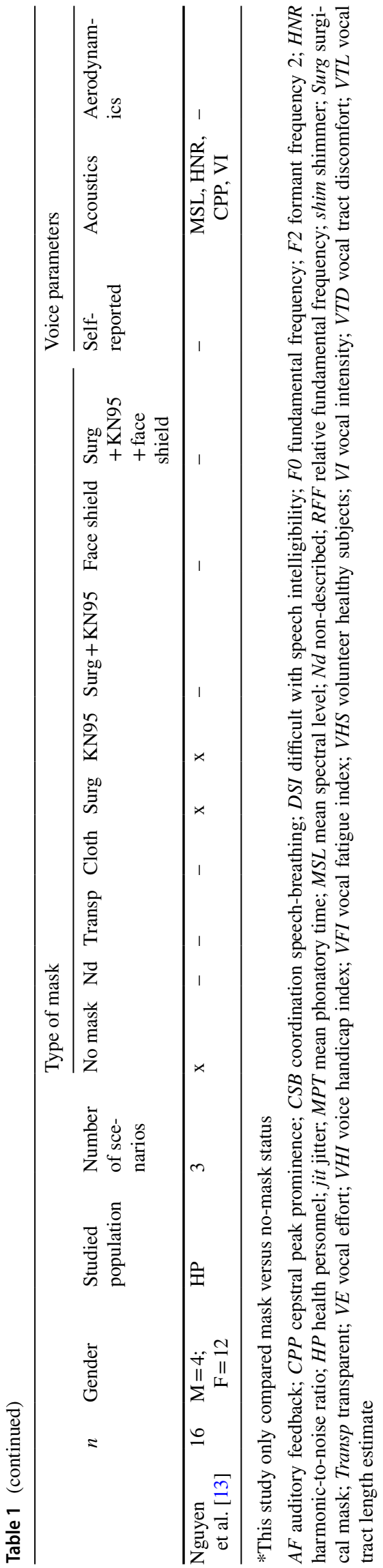

in frequencies below $3000 \mathrm{~Hz}$. Besides, it was observed a significant main effect of masks on speech mean spectral values in high-frequency levels $(1000-8000 \mathrm{~Hz})$ in one study $(p=0,001)$, with attenuation of spectral levels for high frequencies calculated as $5.2 \mathrm{~dB}$ for KN95 $(p=0,005)$ and $2.0 \mathrm{~dB}$ for surgical mask $(p=0,014)$ [13]. This effect was not observed for frequencies below $1000 \mathrm{~Hz}$.

Unlike the other reports on acoustic measurements, Fiorella et al. [10] did not find any significant differences in acoustic parameters between wearing- and not-wearing mask scenarios.

\section{Aerodynamics}

In one of the studies on voice aerodynamics, it was observed a statistically significant difference between Maximum Phonatory Time (MPT) in the mask-wearing group over 45 yearold and the group under 45 year-old $(p=0,032)$ [8]. In the other study, no changes were seen regarding MPT while wearing a face mask [10]. Neither phonatory quotient nor mean airflow rate or mean subglottic pressure was measured in the analyzed reports.

\section{Discussion}

This report presents the first literature review on the potential effects of face masks on voice. The articles included were published quite recently, and describe different populations, methodologies and scenarios. We believe this is going to be an increasingly investigated area, given the sudden need for the widespread use of face masks to achieve the control of SARS-CoV-2 dissemination, and mainly because the current pandemic scenario will probably make mandatory the use of face masks for a long time.

Voice function is multidimensional, so its assessment allows voice quality evaluation in its diverse dimensions, such as self-reported, perceptual, acoustic and aerodynamic [4].

\section{Self-reported voice assessment}

Self-perception questionnaires allow the quantification of how much impact a voice disorder has on the subject's quality of life.

In the analyzed reports, it was verified a significant increase in perceived vocal effort while wearing a face mask $[6,9,11,12]$. The use of a face mask increased the tiredness and voice impairment, as well as the avoidance of voice use, showing significantly higher scores of self-perceived vocal fatigue, a primary perception signal of vocal effort [6]. Also, vocal tract discomfort symptoms were observed to be more frequent and intense while using a face mask. 
This subjective parameter is in straight association with the perception of vocal fatigue and effort and can represent significantly impaired vocal functionality [14]. These symptoms are highly correlated to the need to increase voice intensity to overcome the physical barrier of a face mask [15]. Therefore, an increased risk of hyper-functional dysphonia in mask wearers, especially those who use them in working environment for long periods, should be considered, and modifications in vocal behavior, as well as proper voice training, should be encouraged to prevent eventual future voice pathology. Also, Heider et al. demonstrated mean values of VHI-10 of 7.92 (95\% CI 6.98-8.85) in Chilean healthcare workers, as well as a prevalence of voice impairment of $26.24 \%$. This can demonstrate that healthcare workers and all other subjects that work with face masks can be at a higher risk of voice disorder development, making these authors raise the question of whether this subjects should be considered occupational voice users [12].

The reviewed studies also pointed out that speech intelligibility was significantly impaired with the use of face masks, $[6,9,11]$. This can be explained by the mask attenuation effect, which mainly affects the high frequencies, with a loss depending on the type of mask used. Furthermore, the use of face masks precludes the visual lip-reading and facial expression strategies to assist speech comprehension, which reduces even more speech intelligibility and precludes auditory feedback, due to a reduced vocal monitoring sense.

It must also be outlined that masks can lead to articulatory limitations and restrict the projection of voice sound in space. This attenuation effect on sound was previously described as being of 3-4 $\mathrm{dB}$ for surgical masks and up to $12 \mathrm{~dB}$ for KN95 [1]; the most affected frequencies are 2000-7000 Hz, which are important for speech intelligibility. This attenuating effect of mask appears to depend upon the level of fitting of the masks besides its filtering performance, with speech transmission index values changing about 3-4\% from no-mask condition to surgical masks, and about $13-17 \%$ to KN95 [16]. In another recent study, surgical masks showed to account for $23.3 \%$ of loss in speech intelligibility in a noisy background, a value that can achieve $69.0 \%$ for advanced protection equipment (FFP3 mask combined with face shield). [17].

\section{Acoustics}

The described alterations in the self-perceived vocal characteristics while wearing a face mask were accompanied by some modifications on acoustic voice parameters. These have the main advantage of describing the voice objectively.

Overall, when comparing mask and no-mask scenarios (regardless of the type of mask), it was observed a significant increase in vocal intensity while wearing a face mask in all $[5,7,8,11]$ but two of the six studies performing acoustic voice evaluation $[10,13]$. Intensity reflects the amplitude of vocal fold vibration, and this increase may be due to a compensatory behavior in response to the difficulties being heard. Indeed, vocal intensity is known to be associated with vocal effort, in response to excessive vocal loading [18-20]. People wearing face masks showed a tendency to increase their vocal intensity to make their voices sound clearer [21].

Increased HNR has been observed in speakers with increased vocal intensity [22]. It is believed to be due to the adaptation process while wearing a mask, which may include an unconscious increase in vocal projection. And, in fact, it was verified that the HNR values were significantly increased with the wearing of a mask, either a surgical or a KN95, when compared with the no-mask condition $[8,13]$; it was also increased after a mask-wearing workday comparing with the pre-workday value [11]. Remacle et al. [20] also suggested that an increase in HNR could be linked to hyper-functional voice behavior. The increase in HNR values while wearing a mask can be explained by several factors, namely the adaptation effect, consisting of increasing vocal projection to be heard, as well as the filtering effect, leading to attenuation of glottal noise, that typically appears at high-frequency levels [23], more severely impaired by mask barrier [24].

No significant changes in fundamental frequency (F0) values or Cepstral peak prominence (CPP) were observed $[5,7,8,13]$, contrary to what would be expected in response to an increased vocal loading $[18,19]$. This can happen because face masks encompass a unique challenge, with different and still unrecognized barrier mechanisms.

The effects of masks in articulation and voice resonance could explain the changes in formants frequency observed in some of the studies [5, 8]. In fact, formant frequency F2 decreased in males with multiple overlapped mask scenario and increased in females wearing a surgical mask when comparing with other types of masks, while F3 showed to be significantly lower with masks comparing with the nomask scenario. Formant frequency represents the characteristics of articulation and resonance. They are produced by the vocal tract, which extends from the lips to the vocal folds, and can be influenced by several factors, such as vocal tract length, lips closure pattern, tongue volume and position and lowering of the mandible [25]. These changes in formant frequencies seem to result from the differential filtering effects of masks [1]; they may be influenced by the potential involuntary adjustments of vocal tract properties by mask wearers, to be heard [8]. Besides, it is known that attenuation effects of face masks seem to be higher (with more energy transmission loss) in the higher frequencies, generally above $1000 \mathrm{~Hz}$, as seen in previous studies [1, 26, 27]. F2 and F3 are usually detected above $1000-1500 \mathrm{~Hz}$, so masks may cause a transmission loss at these frequencies and explain the encountered changes. 
Perturbation vocal measures, like jitter and shimmer, were unchanged in most reports [7, 10], except the one from Lin et al. [8] that described significantly decreased values in mask wearers, however interpreted as overstated, probably a result from the increase in vocal intensity and from the filtering mask effects.

\section{Aerodynamics}

We must also outline the lack of studies on aerodynamic changes of voice due to face masks. In this review, we find only two reports analyzing MPT in mask and no-mask scenarios, but other parameters, such as phonatory quotient, mean airflow or mean subglottic pressure, were not assessed.

In fact, some authors already showed that inhaling with face masks is associated with inadequate aerial support, generating a pressure drop across the mask; this requires a more considerable inhalation effort by the wearer, which could be exacerbated when using multiple face masks [28]. Besides, Zhu et al. used computational fluid dynamics to evaluate the re-breathed air in the human nasal cavity with KN95masks respirator, showing that such masks trapped respired air within the respirator, which increases the volume of the fraction of re-breathed air during inspiration. This results in above $60 \%$ of respired air reentering the nasal cavity during the consecutive respirational cycle.

Therefore, it should be interesting to investigate in future studies how mean airflow and mean subglottic pressure values change while wearing a face mask.

The main limitation of this review is the low number of included studies, justified by the so-recent COVID-19 pandemic scenario, which led to the implementation of face masks as a prophylactic safety measure against the propagation of SARS-CoV-2; that makes scientific evidence on the subject still scarce and insufficient. Besides, the lack of evaluation of some vocal domains was verified due to the restrictions in individual data collection during the pandemic. Another important limitation is the different methodology used by the several reports, which limits the analysis of the results and, subsequently, its conclusions.

\section{Conclusion}

The results of this review outline the changes in voice characteristics with the use of face masks. While wearing a mask, people show to increase the perception of vocal effort and fatigue, to increase the sense of vocal tract discomfort and to have difficulties in speech and breathing coordination; besides, speaking with a masks can lead to an increase in vocal intensity, a higher HNR and to an alteration of the vocal tract length and speech articulatory movements, leading to spectral sound changes, impaired communication and perception. These changes may result from the attempt of the speaker wearing a mask to adjust the phonation characteristics to make his voice sound clearer; and this can represent a higher risk for the speaker to adopt vocal misuse behaviors and to develop voice disorders.

Further research is required to analyze the effects of face masks on voice aerodynamics and to study its long-term effects on the potential development of voice pathology, especially for workers who have to wear a protective mask for long periods. Assuming that mask use is a fundamental measure for COVID-19 pandemic control, it must be continued for a long time, so one should be aware of its potential health consequences.

Funding No funding was received for conducting this study.

\section{Declarations}

Conflict of interest The authors have no relevant financial or non-financial interests to disclose.

Ethical statement This article does not contain any studies with human or animals performed by any of the authors.

Informed consent Informed consent collection is not applicable to this study.

\section{References}

1. Goldin A, Weinstein BE, Shiman N (2020) How do medical masks degrade speech perception? Hear Rev 27:8-9

2. Hampton T, Crunkhorn R, Bhat J et al (2019) The negative impact of wearing personal protective equipment on communication during coronavirus disease. J Laryngol Otol 2020:1-5. https://doi.org/ 10.22541/au.159050338.83886289

3. Solomon NP (2008) Vocal fatigue and its relation to vocal hyperfunction. Int J Speech Lang Pathol 10:254-266

4. Dejonckere PH, Bradley P, Clemente P et al (2001) A basic protocol for functional assessment of voice pathology, especially for investigating the efficacy of (phonosurgical) treatments and evaluating new assessment techniques guideline elaborated by the committee on phoniatrics. Eur Arch Oto-Rhino-Laryngol 258:77-82

5. Joshi A, Procter TKP (2021) COVID-19: acoustic measures of voice in individuals wearing different facemasks. J Voice. https:// doi.org/10.1016/j.jvoice.2021.06.015

6. Ribeiro V, Dassie-Leite A, Pereira E, Santos A, Martins P, Irineu $R$ (2020) Effect of wearing a face mask on vocal self-perception during a pandemic. J Voice. https://doi.org/10.1016/j.jvoice.2020. 09.006

7. Magee M, Lewis C, Noffs G et al (2020) Effects of face masks on acoustic analysis and speech perception: implications for peripandemic protocols. J Acoust Soc Am. https://doi.org/10.1121/ 10.0002873

8. Lin Y, Cheng L, Wang Q, Xu W (2020) Effects of medical masks on voice assessment during the COVID-19 pandemic. J Voice. https://doi.org/10.1016/j.jvoice.2021.04.028 
9. Karagkouni O (2021) The effects of the use of protective face mask on the voice and its relation to self-perceived voice changes. J Voice. https://doi.org/10.1016/j.jvoice.2021.04.014

10. Fiorella ML, Cavallaro G, Di Nicola V, Quaranta N (2021) Voice differences when wearing and not wearing a surgical mask. J Voice. https://doi.org/10.1016/j.jvoice.2021.01.026

11. Mckenna VS, Patel TH, Kendall CL, Howell RJ, Gustin RL (2021) Voice acoustics and vocal effort in mask-wearing healthcare professionals: a comparison pre- and post-workday. J Voice. https:// doi.org/10.1016/j.jvoice.2021.04.016

12. Heider CA, Alvarez ML, Fuentes-lópez E et al (2020) Prevalence of voice disorders in healthcare workers in the universal masking COVID-19 era. Laryngoscope. https://doi.org/10.1002/lary.29172

13. Nguyen DD, Mccabe P, Thomas D et al (2021) Acoustic voice characteristics with and without wearing a facemask. Sci Rep. https://doi.org/10.1038/s41598-021-85130-8

14. Badaró F, Araújo R, Behlau M (2014) Vocal discomfort in individuals with cervical complaints: an approach based onselfassessment questionnaires. Audiol Commun Res 19:215-221

15. Guzman M, Castro C, Testart A, Muñoz DGJ (2013) Laryngeal and pharyngeal activity during semioccluded vocal tract postures in subjects diagnosed with hyperfunctional dysphonia. J Voice 27(06):709-716

16. Palmiero A, Symons D, Morgan JW III, Shaffer R (2016) Speech intelligibility assessment of protective facemasks and air-purifying respirators. J Occup Env Hyg 13(12):960-968. https://doi.org/ 10.1080/15459624.2016.1200723

17. Muzzi E, Chermaz C, Castro V, Zaninoni M, Saksida A, Orzan E (2021) Short report on the effects of SARS - CoV - 2 face protective equipment on verbal communication. Eur Arch Oto-RhinoLaryngol. https://doi.org/10.1007/s00405-020-06535-1

18. Anand S, Bottalico PGC (2019) Vocal fatigue in prospective vocal professionals. J Voice 352:247-258. https://doi.org/10.1016/j. jvoice.2019.08.015

19. Whitling S, Rydell R, Lyberg AVH (2015) Design of a clinical vocal loading test with long-time measurement of voice. J Voice. 29:13-27
20. Remacle A, Garnier MGS (2018) Vocal change patterns during a teaching day: inter- and intra-subject variability. J Voice 32:57-63

21. Luo J, Hage SRMC (2018) The lombard effect: from acoustics to neural mechanisms. Trends Neurosci 41:938-949

22. Sampaio M, Masson M, Soares M, Bohlender J, BrockmannBauser M (2020) Effects of fundamental frequency, vocal intensity, sample duration, and vowel context in cepstral and spectral measures of dysphonic voices department of speech-language and hearing sciences, Institute of Health Sciences, Federal University of Bahia. J Speech Lang Hear 63:1326-1339

23. Shoji K (1991) High-frequency components of normal voice. J Voice 5:29-35

24. Madill C (2019) The impact of nasalance on Cepstral peak prominence and harmonics-to-noise ratio. Laryngoscope 129:299-304

25. Pellicani AD, Fontes AR, Santos FF, Pellicani AD, Aguiar-ricz LN, Paulo S (2017) Fundamental frequency and formants before and after prolonged voice use in teachers. J Voice. https://doi.org/ 10.1016/j.jvoice.2017.04.011

26. Corey RM, Jones U, Singer AC (2020) Acoustic effects of medical, cloth, and transparent face masks on speech signals. J Acoust Soc Am 148(4):2371-2375. https://doi.org/10.1121/10.0002279

27. Giuliani N. (2020) For speech sounds, 6 feet with a mask is like 12 feet without. ASHA Lead 26-7

28. Lei Z, Yang J, Zhuang Z, Roberge R (2013) Simulation and evaluation of respirator faceseal leaks using computational simulation and evaluation of respirator faceseal leaks using computational fluid dynamics and infrared imaging. Ann Occup Hyg 57(4):493506. https://doi.org/10.1093/annhyg/mes085

Publisher's Note Springer Nature remains neutral with regard to jurisdictional claims in published maps and institutional affiliations. 